\title{
Life Course Research Agenda (LCRA), Version 1.0
}

\author{
Neal Halfon, Christopher B. Forrest, \\ Richard M. Lerner, Elaine M. Faustman, \\ Ericka Tullis, and John Son
}

\section{Introduction}

A rapidly growing number of research studies show that experiences and exposures in the early

\footnotetext{
Authors' note: In 2010, the Maternal and Child Health Bureau of the Health Resources and Services Administration created the Maternal and Child Health Life Course Research Network (LCRN) and charged it with creating a new infrastructure for catalyzing progress and enhancing funding to support basic, theoretical, applied, and translational life course health development research of relevance to practice and policy. In addition to undertaking a variety of other activities and projects aimed at advancing the emerging field, the LCRN initiated an inclusive agendasetting process that resulted in the Handbook of Life Course Health Development, including this chapter and the research agenda it contains. For a more detailed description of the development and history of the LCRN, the Handbook, and the research agenda, please see the Preface and Introduction to this volume.
}

N. Halfon $(\bowtie)$

Department of Pediatrics, David Geffen School of Medicine, UCLA, Los Angeles, CA, USA

Department of Health Policy and Management, Fielding School of Public Health, UCLA,

Los Angeles, CA, USA

Department of Public Policy, Luskin School of Public

Affairs, UCLA, Los Angeles, CA, USA

Center for Healthier Children, Families, and

Communities, UCLA, Los Angeles, CA, USA

e-mail:nhalfon@ucla.edu

\section{C.B. Forrest}

Applied Clinical Research Center, Children's

Hospital of Philadelphia, Philadelphia, PA, USA years can influence health development throughout life. This research also shows that suboptimal experiences and higher rates of early risk exposures can result in a cascading array of poor health outcomes, with compounding effects across subsequent life stages. This emerging life course health science has fundamentally challenged older models of how health develops and how diseases manifest over the lifecourse. It has also challenged thought leaders, researchers, policymakers, and service providers to redefine the role of health programs and policies as a platform to organize strategies that improve immediate health outcomes for children and their mothers, while also enhancing the health development of the adults those children will become (Wise 2016).

R.M. Lerner

Tufts University, Medford, MA, USA

E.M. Faustman

Institute for Risk Analysis and Risk Communication, Department of Environmental and Occupational Health Sciences, School of Public Health, University of Washington, Seattle, WA 98105, USA

E. Tullis • J. Son

Center for Healthier Children, Families and

Communities, UCLA, Los Angeles, CA, USA 
Life course health science research is "connecting the dots" between child health development, adult patterns of premature morbidity and mortality, and more integrated notions of healthy aging. It is also starting to inform new ways of controlling costs by limiting the prevalence of chronic health conditions. One indication of how fertile, important, and interconnected this research is becoming is the growing number of research studies that are spawning in this nascent field. There are not only many outstanding questions about the relationship between early experiences and lifelong health and well-being, but a growing need to understand how emerging knowledge can be applied to the development of evidence-based practice and policy that can reduce risks, minimize exposures, and optimize lifelong health. As major national research initiatives advance clinical translational research and precision medicine, the life course health sciences can play an important role in informing research questions, leveraging life course informed health development focused strategies, and advancing translational activities from laboratories to patients to communities. Life course health science can play an important role in pinpointing key molecular leverage points, linking those processes to higher-level (body system, organism, family, or social) influences, and illuminating the often overlooked relationship between the individual and population manifestations of underlying health development processes. The findings from life course health science research will also shape the organization, design, and financing of emerging and future healthcare systems (Halfon et al. 2014a, b).

Although this Handbook is far from comprehensive, in that there are numerous health conditions and issues it does not address, it does offer state-of-the-art reviews and analyses of life course health development research focused on key life stages and a variety of specific health conditions. Based on their analysis of existing research, the authors of each chapter have suggested how life course health development research can be supported and advanced. In developing this concluding chapter, we synthesized their findings and recommendations in order to propose an integrated set of research priorities and strategies that can advance the entire field of life course health development research.

The resulting transdisciplinary research agenda focuses on foundational research types and topics, including research that addresses life course epidemiology, research that elucidates life course health development (LCHD) processes (e.g., sensitive periods, epigenetics), and research that identifies effective interventions, with a special emphasis on health disparities research and research on high priority conditions (e.g., obesity and mental health conditions) and specific vulnerable populations (e.g., children in foster care, premature infants, and children with medical complexity). Next, we consider essential research capacity-building activities focused on data, data systems, and methods, as well as strategies for building the human, technical, cultural, and financial capacity required to carry out this challenging work. Lastly, we discuss the importance of translational and intervention research that can speed the application of concepts and findings to the practice and policy arenas. We conclude with a discussion of advancing the overall life course health development research paradigm, including the framework and principles that have emerged from the research thus far, and that are helping to define a robust explanatory and theoretical LCHD framework.

\section{Rationale: Why a Life Course Research Agenda?}

Until recently, a strong US-based life course focused research and data infrastructure has been lacking. There have also been limits on funding currently available in the US to support the development of new methodologies and collaborative approaches to life course health development research. This has hampered the production of the transformative research that is needed to advance the emerging field of life course health development. The recently aborted National Children's Study is a major example of an unsuccessful attempt to launch a cohort study and research infrastructure that would have 
allowed the US to start catching up with the many other nations that have launched one or more national cohorts in the past, are in the process of conducting millennium cohort studies, or already have well developed national data systems that permit exploration into life course specific health development questions. Nonetheless, the move toward multidisciplinary LCHD research is advancing in the U.S. as research continues to break out of discipline-specific silos that have often constrained rapid transdicsiplinary progress. Over time, we believe that life course health development will become an increasingly productive and impactful transdisciplinary field.

Current and future production, dissemination, and application of life course health development research can improve our understanding of the origins, development, and manifestation of health and disease. This research must also be strategically aligned with and responsive to ongoing and emerging trends that are important influences on health development research. Because the epidemiology of human health and the capacity of the health system to respond to challenges are constantly changing, a robust LCHD research strategy must be informed by and responsive to emerging trends and shifts. These include but are not limited to:

- The ongoing epidemiologic shift in diseases and disease burdens, from infectious diseases of the past to chronic, preventable, noncommunicable diseases.

- The recognition that health is not the absence of disease, and that health as an outcome is a key target for healthcare systems.

- The "new" epidemics of obesity, several mental health conditions, and multi-morbidity, particularly in older adults.

- The growing recognition that contributing to the US's dubious distinction as the sickest of rich nations is the fact that it spends more on healthcare late in life, compared to nations with better health outcomes that invest in social and education services early in the life course.

- The World Economic Forum's recent focus on what they have termed as "inflection points" across the life course that serve as strategic targets for intervention and present opportunities to leverage population health improvement goals. These inflection points of health development suggest that life course health development research can be translated into effective policy frameworks.

- The transition in how healthcare services are organized and paid for, including the focus on preventing disease and designing payment methodologies that pay for health instead of services. These major system-level changes can use the new science of health development to define the value of healthcare, align payments with health development time frames, and establish overall health system goals.

- A range of different research organizing strategies-from those focused on translating research into action at a patient, community, or health system level to the Clinical Translation Science Institutes, to the National Institutes of Health's new efforts focused on precision medicine-can potentially benefit from new insights from the life course health sciences and can also help advance this type of basic, clinical, applied, and translational research.

The research revolution that gave birth to the life course health sciences is now about four decades old. Several disciplines, fields, and seminal research studies have contributed to the emergence of the field. These converging, interacting, and interdependent empirical and analytic trends are important to recognize and to strategically leverage as we advance the life course health sciences through a robust research agenda.

Longitudinal cohort studies that have allowed for direct empirical interrogation of the role of early life factors on adult health behaviors and outcomes contributed to the emergence of the field, as did the recognition that the impact of early adversity on lifelong health was not being captured by prevailing biomedical and biopsychosocial models that ignored the timing and influences of social and environmental factors on health development. David Barker et al.'s (1989) pathbreaking research describing the long-term 
associations between perinatal health conditions, such as birthweight, and adult chronic disease decades later provided an important empirical nudge for considering a new form of life course epidemiological research.

More recently, chronic disease epidemiologists have teamed up with neurobiologists and molecular biologists to elucidate the biological mechanisms for the effects of early childhood exposures and experiences on later and lifelong health. While these largely biomedical research studies were being done, a parallel set of investigations in sociology and psychology examined how mental health develops over the life course in response to interpersonal and environmental interactions. The convergence of life course epidemiology, biology, life course sociology, and life span human development psychology has established the contours of a multidisciplinary field of life course health development (Kuh and Ben-Shlomo 2004; Ben-Shlomo and Kuh 2002; Halfon et al. 2014a) that is in the process of maturing into a unified, transdisciplinary field with a unique set of theoretical constructs, frameworks, models, and methodologies.

By integrating evolutionary and developmental perspectives, the life course health development framework also spans the gap between these two fields of research that emerged with the post-Darwinian synthesis of evolution and heredity, coupled with advances in human genetics and epigenetics. As the LCHD framework began to take shape, it helped support the growing recognition that (1) health development is a complex adaptive process that emerges out of the dynamic coactions between an organism and its environments, (2) this emergent process is mediated by different types and levels of plasticity that evolution has engineered into the human developmental process to optimize a range of adaptive responses to diverse environments, and (3) the synchronization and harmonization of different embedded time frames must be considered, assessed, and understood (Hochberg 2009).

LCHD as a discrete, multidisciplinary field of inquiry is young. Irrespective of how conceptually attractive constructs such as sensitive periods or biological conditioning might be, it is important that the field be appropriately self critical in determining how strong the evidence really is for these notions. The biological plausibility of many of the LCHD hypotheses about the role and impact of different types of plasticity on the development of health and disease needs to be tested and strengthened by carefully designed and implemented research. The tension between advancing the field and ensuring sufficient awareness and reflection about whether and how it challenges the prevailing orthodoxy of biomedical research requires more careful analysis.

Hanson and Gluckman have highlighted the reasons behind the delayed acceptance of the concept of the Developmental Origins of Health and Disease (DOHaD), including a lack of conceptual framework, confusion between factors correlated with disease risk and those involved with causation, assumptions that all of the $\mathrm{DOHaD}$ effects were operating only through the pathway of lower birthweight, lack of plausible biological mechanisms, failure to recognize the importance of the $\mathrm{DOHaD}$ concept under normal developmental conditions as opposed to extreme conditions, lack of evidence of its relative importance in relation to other risk factors (i.e., population attributable risk analysis), and lack of plausible ways to use the concept clinically and in public health (Hanson and Gluckman 2014). Many of the barriers that Hanson and Gluckman describe are part of the impetus and genesis for further developing the LCHD framework so that DOHaD concepts - including notions of sensitive periods, biological priming and conditioning, life histories, and evolutionary constraints - can be used to further empirical, analytic, translational, health services, policy, and conceptual research.

The LCHD framework that has been articulated in this volume by Halfon and Forrest (2017), while not a fully developed paradigm, provides a new approach for linking health and developmental processes. The framework challenges the explanatory power of older and more linear biomedical models, as well as multidimensional biopsychosocial approaches. The LCHD framework is portrayed as a dynamic network of interactive and interdependent concepts that are coming of age 
and formulating this new investigative approach. In spite of the challenges for moving life course health research forward, the amount of consistent evidence, the convergence of several fields of inquiry, and the recognition of common principles are providing the opportunity - and need - for not only a more coherent explanatory framework but for the second generation of priority research topics, questions, activities, and strategies that are presented in the next section.

\section{Recommendations}

The following recommendations are presented in four main sections: (1) priority research types and topics, (2) priority research capacity-building activities, (3) translational priorities pertaining to the application of current knowledge to practice and policy, and (4) strategies for refining the LCHD theoretical framework.

\subsection{Section I: Priority Research Types and Topics}

\subsubsection{Priority Research Types Epidemiological Research}

There is a growing recognition of the value of life course chronic disease epidemiologic research and its application to understanding the origins, distribution, and patterns of various diseases and health conditions. LCHD epidemiology has benefited from a better appreciation for the timing and time-specific interactions of numerous multilevel factors that influence health development. As the importance of what has been called the exposome (i.e., the set of environmental factors that influence health) become more salient and new measures and approaches for assessing environmental influences are created, a demand will be generated for multilevel epidemiologic studies (Robinson et al. 2015). Better epidemiologic measures, modeling, and analysis will all be needed to understand how different kinds of adversity interact with one another and impact health development (see Buka et al. 2017; Little 2017).
A better, more systematic, and useful understanding of how social adversity stimulates different stress responses, how the development of those stress reactivity patterns are biologically conditioned by other stimuli, and how they cascade, or play forward, requires studies using longitudinal cohorts. Such studies will need to further refine the characterization and measurement of different and specific kinds of environments. This includes better measurement of specific components, experiences, and exposures but also how different elements of that environment are contextually linked and expressed both independently and synergistically. Understanding the interplay of genes and environment, and how different contexts influence the emergence of different trajectories, requires that epidemiologic studies focus on proximal neurobiological processes, including the epigenetic influences on the emergence of behaviors that manifest as endophenotypes and how environmental context influences the progression to full-fledged behavioral or psychiatric disorders (see Boyce and Hertzman 2017). This raises a number of measurement challenges, especially concerning how to assess changes in interacting and interdependent influences from the cellular to the social level.

A new fruitful area of LCHD epidemiologic research will focus on better characterizing the role and impact of the places where children and families live, grow, learn, work, and play (Pearce 2014; Dunn et al. 2015). With the expanding number of place-based interventions that attempt to improve the organization and functioning of entire community ecosystems, there is a mounting interest in how place as an organizing force influences health development at both the individual and population levels. The notion that multiple risk and protective factors are uniquely organized and deployed by a particular place provides new ways of understanding how to design and implement place-based interventions that strategically aggregate services, programs, and systems into a more coordinated approach to optimizing health development (Pearce et al. 2016). 
Given the role that different mediators and modifiers can play at different ages and with different subgroups, more research is needed to determine how both normal and disordered development unfolds in relationship to nested exposures and experiences. Such research should also include a more strategic focus on how mutable these relationships might be. Specific interventions have been developed to modify family relationships, including interventions focused on parent-child relational content, maternal and infant bonding, or the capacity of mothers and fathers to co-parent (Leslie et al. 2016). How these interventions transform the ecosystem in which the child is developing, and how these alterations track over chronological and developmental time frames, requires further study.

While developmental trajectories are routinely used by developmental psychologists to describe the patterns and contours of developmental pathways and how they change over time, the measurement of health development trajectories in relationship to different proximal (maternal depression) and distal (violent neighborhood) risks, as well as different proximal (supportive grandmother) and distal (strong faith community) protective factors, is sorely lacking. A better understanding of how these different risk and protective factors interact and influence, both individually and in combination, health development requires different measurement and study design strategies than are routinely incorporated into cohort studies where exposures are evaluated as independent influences and causal attribution is often oversimplified (Dunn et al. 2014).

LCHD epidemiologic studies should also focus on better characterization of the role that differential timing of exposures to risk and protective factors has on the emergent quality and function of developmentally contingent hormonal systems and on the development of superordinate behavioral capacities including executive functioning, empathy, and perseverance. In addition, there is also a great need to focus on how multitiered pathways emerge, how they are influenced by different interventions, and how those influences affect outcomes over years and decades.

\section{Mechanism Research}

As the life course health sciences mature, they are more fully embracing the complexity of health development. There is a growing realization that health development is a complex adaptive nonlinear, multilevel, and multidirectional process in which the timing, patterning, and interactions of exposures and experience play out against a set of evolutionarily influenced developmental processes, each with its own sequencing and time signature. Throughout this volume, the need for better and more precise mechanism research has been emphasized, such as:

- Elucidating the nature and role of, and the mediators that influence, how sensitive periods emerge, develop, and manifest

- Developing a better understanding of how family- and community-level factors interact and influence each other over time

- Evaluating how the health capital that mothers bring to conception influences prenatal as well as postnatal child health development

- Assessing how biological sensitivity to context emerges and is modified by different signals and cues from both slowly and rapidly changing environmental influences

Biological conditioning-also sometimes referred to as biological priming or, more deterministically, biological embedding - includes the various ways in which environmental influences either exert transitory or more long-lasting impacts on developing biobehavioral regulatory systems. This is a high-value target for future research. Key questions include: What are the specific biological, neurologic, epigenetic, and physiologic mechanisms that are most important for altering biobehavioral regulatory systems? What is the mutability of these mechanisms? Do certain key factors act as "triggers," setting in motion a cascade of future biological response patterns? Biological conditioning processes can range from the exposure to a specific nutrient like folate and its influence on epigenetic methylation based on availability in indigenous diets (see Herman and Taylor Baer 2017) to the role that different patterns of sleep and sleep duration have 
on cycling of neuroendocrine hormones that serve to prime other factors related to growth, metabolism, and the biological underpinning of behavioral response systems (see Mummert et al. 2017; Hawkins et al. 2017).

Given worldwide rising rates of neurological, mental health, developmental, and substance abuse (NMDS) disorders, the international health community has prioritized developing a better understanding of the epigenetic effects of nutrition, infections, a range of environmental exposures, and psychosocial factors on developing nervous systems, especially the role early exposures and experiences have on later onset neurological conditions (Silberberg et al. 2015). Included in this prioritization is the recognition that adolescents are particularly at risk for developing neurocognitive deficits and mental health problems. Given that $50-75 \%$ of lifetime mental health disorders have their onset before age 24 , a focus on better understanding the mechanisms that create this long-lasting vulnerability and how it can be addressed is also a high priority (Davidson et al. 2015). The life course health sciences should capitalize on worldwide interest in NMDS by highlighting the importance of life course approaches to understanding how NMDS develops (Birbeck et al. 2015).

Several chapters in this book emphasized the role of biological and behavioral transitions as sensitive periods and how they may afford leverage to alter developmental trajectories (see DelGiudice 2017; Boyce and Hertzman 2017; Wood et al. 2017). Similarly, interactions between the hypothalamic-pituitary-adrenal axis and basic energy metabolism that are also controlled by the hypothalamus may be relevant to the development of both physical and mental health, and understanding these interactions holds promise in better understanding why conditions like attention-deficit hyperactivity disorder (ADHD) and obesity cluster together (Halfon et al. 2013).

The way in which one sensitive period emerges and influences the development of subsequent sensitive periods also bears further investigation. This includes how the preconception period serves as an important platform for the creation and management of health development capital, and how the preconception period organizes and influences the prenatal and subsequent postnatal periods, each with its own defining characteristics, functions, and sensitivities. As more attention focuses on the prevention of adverse birth outcomes and the promotion of equity from the start of life, our understanding of the role and function of the preconception and prenatal periods in lifelong health will become all the more important. As capacity improves to identify, measure, differentiate, and understand the relationships between different sensitive periods of health development, our ability to devise and test more longitudinally integrated healthoptimizing interventions will also assume greater importance.

Many of the chapters emphasized the need for research designs that specifically target multilevel influences, multilevel measurement, and analytic techniques that focus on multilevel interactions and modifications. They also suggested extant data limitations and methodological challenges that pose barriers to measuring and modeling the complexity of these influences and interactions. A whole new set of analytic tools and modeling techniques are emerging from the world of complex systems science. Their adaptation and application to the complex multilevel and life course health development processes is likely to be very fruitful and illuminating (Osypuk 2013; El-Sayed et al. 2013).

Evolutionary perspectives on how adversity, stress, and complex evolving cultural structures (e.g., educational and religious institutions) have shaped selection of human development priorities are also ripe for further cross-disciplinary research. The chapters by Boyce and Hertzman (2017) and DelGiudice (2017) in this volume emphasize the importance of how environmental stressors can lead to diverse adaptive responses that can manifest at different levels of impact depending on other contextual factors. They suggest that in advancing life course health development research, we recognize the value of cross-species, comparative studies that can shed light on the relationship between evolutionary biology and social environments and their conse- 
quences on human developmental adaptation and environmental mismatches.

Lastly, the evidence reviewed in several of the chapters suggest the potential value of a more integrative developmentally nuanced systems-physiology approach to understanding how co-occurring risks manifest in developmentally comparable changes across multiple physiological systems (Singer and Ryff 2001). For example, while it is well established that exposure to aminoglycoside antibiotics early in life can have detrimental and potentially devastating effects on both auditory and renal function, the chapters in this volume by Russ and colleagues (Russ et al. 2017) on hearing and Brophy and colleagues (Brophy et al. 2017) on chronic kidney disease (CKD) suggest that there are ways that the developing sensory apparatus in the ear and the developing filtration apparatus in the kidney may share other potential vulnerabilities across the life course. Future research on how risks and protective factors influence multiple systems will potentially lead to multi-solving interventions that can optimize health development across several discreet physiological systems. Better modeling of how seemingly distinct biological and behavioral systems develop holds promise to understanding how they develop their inherent robustness, where they share similar vulnerabilities, and how they might be buffered and protected from undesirable insults and risks (Kitano 2004; Cohen 2016).

\section{Intervention Research}

Our understanding of the impact of life course risks will be improved by better intervention research that targets key developmental processes and time periods. Throughout this volume, authors have highlighted the important role of intervention research for better understanding not only how to intervene, but also when. For example, the chapter by Hawkins and colleagues (Hawkins et al. 2017) suggests that a key to reducing childhood obesity is finding the right level and time in the life course to intervene for the maximal effectiveness and efficiency of interventions. However, the challenge of mounting programs designed to modify obesogenic health development pathways may be quite complex, involving multiple settings (e.g., medical care, homes, child care, school) (Taveras, Blackburn, et al. 2011a; Taveras, Gortmaker, et al. 2011b; Taveras et al. 2012), or components (e.g., system redesign, individual behavior change strategies including e-technology) (Lubans et al. 2010; Taveras et al. 2012), and targeting single or multiple factors (Taveras, Gortmaker, et al. 2011b; Vesco et al. 2012).

The chapter in this volume by Kim and colleagues (Kim et al. 2017) highlights how new insights into multilevel influences on health development pave the way for a range of different interventions that will further clarify these relationships. New research on pregnancy and the first years of a child's life indicate not only that this is a sensitive period for child neurological development but that it is a period when a parent's brain may demonstrate higher levels of plasticity and undergo changes to support the parental role (Kim et al. 2016). Given that parental brains are being remodeled at the same time that children's brains are developing, there are several potential ways to intervene so that parents modify home environments in developmentally optimizing ways for both their children and for themselves. Many potential interventions that span from cells to society have been studied in isolation, ranging from those that attempt to modify socioeconomic position via cash transfers to those that focus on harmonizing parenting approaches via co-parenting skills training (see Kim et al. 2017; Duncan et al. 2011; Feinberg et al. 2009). Less research has been done to better understand how best to combine interventions into a more integrated, sustained, and sustainable developmentally optimizing strategy.

The chapter in this volume by Boyce and Hertzman (2017) suggests that more effective prevention science demands new and innovative intervention designs that are responsive to the emerging science of health development. Highlighting how the Nurse Family Partnership broke new ground when it was first conceived and launched, the authors point to the need for 
ambitious and "more radical departures ... from the conventions of traditional early development programs, including careful consideration of how complex dynamic systems thinking might be wed to novel, preventive interventions."

The chapter in this volume by Larson and colleagues (Larson et al. 2017) similarly focuses on the importance of intervention research to better understand the origins, impact, and mutability of health disparities that arise from different health development pathways. They argue that a multilevel intervention research strategy is needed. Intervention research on decreasing disparities in health development could focus on a range of possible sensitive periods, examining the possible and potential benefit of and best timing for interventions in the preconception period for improving birth. They also suggest that new social interventions, designed to alter specific short-term health outcomes (e.g., asthma or early stress reactivity) in at-risk populations, should be designed and tested in relation to how they modify long-term health and well-being outcomes in adulthood. For example, can population-level interventions like teaching young children yoga and other mindfulness techniques serve to "inoculate" them against elevations in allostatic load due to stressful and chaotic family environments?

Questions about the best timing for intervention are important. Cost-effectiveness studies can be used to compare the value of interventions at different life stages. In addition to interventions at the individual level, interventions focused on the multilevel determinants of health (e.g., neighborhood-level interventions) will also be important. Lastly, the chapter by Larson and colleagues (Larson et al. 2017) suggests that research should focus on the impact of new policies and programs at the local, state, and national levels to reduce health disparities across the life course. The authors also make an important distinction between research focused on minimizing risk and preventing poor health and interventions aimed at optimizing health and developmental potential.

\subsubsection{Priority Research Topics}

\section{The Impact of Adverse Experiences on Health Development and the Origins and Implications of Health Disparities}

Because of growing and persistent income inequality, high rates of child poverty, and associated levels of adversity, there is a pressing need to better understand the impact of adversity on health development, as well as ways of mediating and modifying its influence. Recent data from the National Survey of Children's Health reveals that the experience of adversity is not confined solely to children living below the federal poverty level, but extends up the income ladder (Halfon et al. 2014c). With nearly half of all children living in low income families with earnings below $200 \%$ of the Federal Poverty Level, these are concerns that now affect a majority of children. Related to the greater prevalence and experience of adversity in children from low income families is the relationship that the experience of adversity has to the development of a range of phenotypic responses.

Research on childhood adversity has been highly influenced by the conceptualization of adversity advanced by Felitti et al. (1998) in the original Adverse Childhood Experiences (ACE) Study. This retrospective cohort study of patients attending a weight reduction clinic at a southern California Kaiser Permanente facility in the 1990s has provided important information on the relationship between adversity early in life and the subsequent occurrence of a range of chronic diseases. However, it has also limited the conceptualization of adversity and how it is measured. Moving forward, it will be important to refine our definition and measurement of adversity, as well as the patterns and variations in its occurrence. The recognition and description of how specific types of adversity can be traumatic, and how that trauma can in turn lead to different kinds of stress response patterns, also call for a better understanding of the relationship between adversity, trauma, and stress. Given that all adversities are not equal, and that not all adversity is experienced as trauma, it is important to develop better measures of the experience of adversity, as well as to examine how that adversity is transduced by 
the brain and other body systems, including behavioral response patterns, proximal neurobiological processes, and epigenetic and other molecular changes (Hane and Fox 2016).

The chapter by DelGiudice (2017) in this volume warns that researchers should exercise caution in assuming that undesirable developmental outcomes reflect dysregulation of biological systems, and should remain open to the possibility that those outcomes may be part of adaptive (or formerly adaptive) strategies for survival and reproduction. He argues here and elsewhere (Del Giudice 2014b, c) that a life history framework is especially useful in teasing out the logic of potentially adaptive combinations of traits, highlighting critical factors in the environment, and bridging behavioral development with physical growth trajectories. A similar observation is made in the chapter in this volume by Lerner et al. (2017) in their analysis of adolescent health development, highlighting the context dependency of adaptive developmental regulation and how such regulation may vary by specific social situations, times, and places.

Related to the experience of adversity is the capacity for resilience among individuals and populations that experience it. The chapter by Larson and colleagues (Larson et al. 2017) suggests that additional research is needed to not only identify individuals with exceptional health resilience but to better understand the health development pathways that lead to more resilient phenotypes. Studies that examine the cultural, social, and psychological resources that can lead to thriving and better-than-expected health outcomes for individuals from disadvantaged social groups should also receive priority consideration, along with studies that lead to a better understanding of how those resources affect entire populations.

A basic notion of the life course health development framework is that the balance and interplay of risk and protective factors influence health development trajectories. Children living in environments with many more risk factors than protective factors will have a greater propensity for delayed or disordered health development trajectories, while those growing and developing in environments with more protective and healthpromoting factors will achieve more optimal developmental pathways and trajectories. However, this simple characterization of the distribution of risk and protective factors in lowversus high-income communities obscures more complex and dynamic processes, a high degree of variability, and other deeper dynamics that might not be readily apparent and need to be considered and measured more directly. As the chapter in this volume by Mummert et al. (2017) points out, the ecosystems in which today's children are developing are novel when conceptualized across evolutionary timescales, with our manmade environments posing additional biological and behavioral challenges to developing humans that can both mediate and exacerbate other forms of more readily observable and measurable adversity.

While the experience of adversity has been directly related to the onset and development of different health outcomes that range from heart disease to depression, the relationships and variability at a population level is less well defined. The chapter in this volume by Larson and colleagues (Larson et al. 2017) suggests that it is important to continue to investigate the underlying biological processes that contribute to health disparities in human populations, and to better understand the role of protective factors and resilience on positive health outcomes for individuals from disadvantaged backgrounds. They also suggest more attention to parental preconception health and other intergenerational mechanisms that may contribute to early health disparities. To inform public policy and further elucidate the link between adversity and health disparities, they suggest additional investigations of the dynamic multilevel contributors to health status disparities across the life course.

\section{The Impact of Family- and Community-Level Factors on Health Development}

The LCHD framework highlights how the importance of understanding how health development processes is influenced by multiple factors at multiple levels in dynamic, transactional ways. Of particular salience to practitioners is the role that family- and community-level factors may 
have on health development. Related to the independent impact of these factors is their combined impact, as well as the impact that comes when these factors are considered in relationship to a particular ecosystem or place. This kind of multilevel and integrative analysis is important in a world where place-based strategies aimed at creating multiple potential opportunities for health improvement are routinely deployed.

Several of the chapters emphasize the importance of multilevel contributors to health status disparities over the life course, with a particular emphasis on upstream structural factors and neighborhood-level determinants. Multilevel studies can help examine the impact of neighborhood-level factors on health disparities by socioeconomic status, race, and ethnicity. Also needed are studies that incorporate longitudinal or life course measures of neighborhood and environmental exposures. At the population level, studies are needed to examine the natural environment, built environment, and social environment exposures that contribute to geographic differentials in rates of health and disease, as well as how variation in health policies contribute to disparities. To the greatest extent possible, research studies that examine lifelong contributors to sociodemographic health disparities should investigate the importance of the timing of key environmental and social exposures.

There is also a need to better understand the role that family and community risk and protective factors play in mediating influences at different levels. For example, how do positive health development enhancing family routines and other family-centric protective factors insulate or buffer a child's response patterns in a community where violence, social chaos, and family isolation are the norm? Evaluating the contribution of family capacities and routines as well as social isolation, social relationships, and social support-is a fertile and strategically important area for further research. This kind of research is particularly important for a healthcare arena in which "care bundles" are being developed to address both the medical and nonmedical social, family, and behavioral influences on health.

\section{The Impact of Specific Exposures and Experiences, the Importance of Various Life Stages, and the Development of Health Conditions}

There are dynamic developmental relationships between different kinds of experiences and exposures that can be better understood from a life course perspective. For example, the chapter in this volume by Lerner and colleagues (Lerner et al. 2017) suggests profiling how different patterns of nutrition develop and influence a range of related behaviors and outcomes during adolescence. How do adolescents' diets influence their cardio-metabolic risk as adolescents and adults? Do adolescents maintain a healthy diet across different settings involving peers and family members? Is there age-associated variation in answers to this question, for instance, variation associated with pubertal development or pubertal status/ stage (e.g., early, on time, or late)? Do answers to these questions vary in relation to gender? As well, do they vary in relation to normative social transitions (e.g., moving from elementary school to middle school)? How do peer social networks influence these behaviors or family mealtime behaviors? Are answers moderated or changed by the socioeconomic, cultural, religious, or national contexts of youth? In turn, do answers here vary in relation to media exposure about eating and about desirable body types that may be prevalent for youth living in particular settings at particular times in history? Given the synchronization issues raised by the seventh life course health development principle (see Halfon and Forrest 2017), how are all these answers moderated or changed by what may be nonnormative life events in the lives of adolescents? Examples here may be the death of a parent or deployment in the military (Cozza and Lerner 2013), family disruption due to divorce or separation, or family challenges in the face of environmental tragedies such as weather.

\section{Important New Epidemics or Emerging Changes in Population Epidemiology}

Rapidly changing environmental conditions and exposures have resulted in a variety of epidemiological transitions that yield new patterns of 
health and disease. Perhaps the most dramatic epidemiologic transition is the one that took place as a result of improvement in sanitation and public health - from mortality due to infectious or communicable disease to mortality due to noncommunicable chronic health conditions like diabetes, hypertension, heart disease, and cancer. This transition has been well described and appreciated, although not without controversy as to its origins and drivers. In the past several decades, we have witnessed increased rates of child obesity and child and adolescent mental health disorders. While there have been relatively simple notions about the origins and drivers of the obesity epidemic, the chapter by Hawkins and colleagues in this volume (Hawkins et al. 2017) highlights the developmental complexity underlying this major epidemiologic change.

There has been far less focus and research on the developmental origins and patterns of manifestation of a range of mental health problems that appear to be rapidly increasing in children and adolescents. Recent epidemiologic surveys suggest that over $20 \%$ of adolescents now have a mental health disorder with impaired functioning (Merikangas et al. 2010). A better understanding of the mechanisms, causal factors, mutability, and lifelong consequences of these disorders is sorely needed. While there has been some research on whether mental health disorders arising in adolescence persists into adulthood, there is evidence that when they do, the impact on functioning and lifelong success can be more debilitating than the lifelong impact of chronic medical conditions (Moffitt 1993; Currie and Stabile 2006). While there have been studies that have begun to link increasing rates of media and television exposures to the development of attentional problems, the nature and impact of this rapidly changing epidemiology calls out for better life course health development research (Zimmerman and Christakis 2007).

\section{The Health Development of Particularly Vulnerable Populations}

The health development of particularly vulnerable populations of children demands more life course focused research. The chapter in this volume by Msall and colleagues (Msall et al. 2017) highlights the impact of socioeconomic-associated risks on the health development of premature infants. While there have been many longitudinal followup studies of premature infants, few have adopted a health development perspective or examined the role that other risk and protective factors play in shaping the developmental trajectories of this very vulnerable population. The same can be said for individuals with autism spectrum disorder, CKD, or most other chronic health conditions. In their chapter on autism spectrum disorder (ASD), Drmic, Szatmari, and Volkmer highlight how little that is known about the trajectories of children and adolescents with ASD and how studies focused on optimizing the health development of individuals with ASD could provide critical information. They highlight how adolescents with ASD who are clearly benefiting from participating in school and other educationally related activities experience a functional downturn once the developmental scaffolding that school provides is no longer available. The suggestion that individuals with ASD have delayed maturation of key developmental processes also argues for a more developmentally attuned and integrated understanding of how to optimize outcomes. The chapter on CKD by Brophy and colleagues (Brophy et al. 2017) also connects the dots between prematurity and other insults that may lead to decreased number of functional kidney filtration units (nephrons) and the time-specific and cumulative insults that are increasingly understood as the life course developmental pathway to CKD.

There are several other vulnerable populations of children where a life course health development perspective could be very illuminating and more importantly could shape the services and interventions that are provided. For example, it is well known that children who are abused, neglected, and placed into the foster care system can experience dramatically altered life course trajectories (Zlotnick et al. 2012; Tyler and Schmitz 2013; Patterson et al. 2015). Initial studies examining the impact of adversity, institutional placement, and out-of-home care have suggested how such experiences can alter neurobiological functioning and cellular processes 
associated with the regulation of cellular aging and longevity (Drury et al. 2012; Karatsoreos and McEwen 2013). Life course health development research could improve these interventions in regard to their capacity to improve long-term trajectories for children in foster care and other vulnerable populations, including young people involved in the juvenile justice system and children with chronic and debilitating medical conditions and impairments (Zlotnick et al. 2012). In each case, better understanding the health, growth, and developmental trajectories - and the factors and experiences associated with optimal health development outcomes - is sorely needed.

\subsection{Section II: Priority Research Capacity-Building Activities}

\subsubsection{Advance LCHD Measurement Capacity and Improve Study Design to Provide Useful Data and Promote Greater Collaboration Across Disciplines}

Throughout this volume, the authors have emphasized the importance of new and better measures of health and health development, with specific reference to developing and aging body systems (e.g., kidney health, hearing health). Closely related to measurement research are new and better analytic strategies that utilize data in more creative ways. Several chapters emphasize the need for better measures of "positive health." For example, Russ and colleagues' chapter on hearing (Russ et al. 2017) suggests that researchers need operational measures of positive hearing health to enable better measurement of treatment outcomes and functional hearing ability. Measures of positive health-including oral health, nutritional health, and mental health - as well as measures of positive health trajectories in these and other areas provide an empirical way of measuring both general and targeted healthoptimizing strategies. Such measures might be composed of several different related measures and biomarkers that could be used to profile a specific health development capacity.
Measures of positive health development are not only important for assessing the health status and trajectories of individuals but also for use in assessing populations. The Early Development Index is a measurement tool that is used in Canada, Australia, and the USA to assess healthy development and school readiness for 4-6-yearolds (Janus et al. 2016). The EDI is composed of 110 items that span 5 dimensions and 16 subdomains of functioning. It is routinely collected and mapped at the neighborhood level to provide a population measure of health development. It has been used to predict early language and cognitive development and to examine differences in cognitive and emotional development in populations of children (Brownell et al. 2016). It is part of an evolving set of population health development measurement tools that now includes the Middle Development Index that was designed to measure similar dimensions in 9-11-year-olds (SchonertReichl et al. 2013; Guhn et al. 2016a). The same Canadian team that created the EDI and MDI is in the process of developing health development indices covering the entire child span, using the EDI to develop a pan-Canadian population health monitoring system (Guhn et al. 2016b). In addition, the EDI has also been used as a measure in "cells to society" research that is examining the impact of social gradients and how that manifests in relationships to social epigenetics (Hertzman and Boyce 2010).

Enhanced data collection of individual behaviors, traits, and biomarkers is now possible using low-cost, readily available technologies like smartphones and self-reported questionnaires. The National Institutes of Health have invested millions of dollars into the creation of state-ofthe science self-reported measures of physical, mental, and social health as part of its program called the Patient Reported Outcome Measurement Information System (PROMIS, see www.healthmeasures.net for more information). These assessments of health can be combined with a wide array of environmental sensor assessments that can measure macro and micro fluctuations in the local environments, as well as clinical data stored in electronic health records and other molecular data such as genetics and epigenetics. 
Aggregation of large amounts of data of these types can be done to create big health development data resources that can be mined and used to elucidate novel, unanticipated associations to inform future causal modeling and interventions.

Advances in life course health sciences have depended on the creation of longitudinal data collection efforts over the past century, most of which have been undertaken in countries located in Europe and Oceania. Recognizing the value of these longitudinal studies, several nations launched millennial cohort studies. This volume profiles several existing longitudinal surveys that can be used for examining life course impacts of different social and environmental factors (see Geller et al. 2017; Sastry et al. 2017; Cooksey 2017).

Longitudinal life course studies are important for capturing the dynamics of intra-individual health development trajectories and their environmental influences and epigenetic profiles. Because longitudinal cohort studies capture temporal relationship between exposures and outcomes, they can assist in defining causality and also in identifying predictive epigenetic biomarkers that may be used clinically and for targeted and more precise public health interventions.

There is also growing recognition that existing longitudinal and disease focused cohorts could be more effectively used to investigate specific questions by increasing sample sizes to enhance statistical power to examine interactions. Work is underway both in the USA and Europe to align and link cohorts, recognizing that the heterogeneity of the different studies will require conceptual and analytic harmonization to allow data to be pooled. Existing cohort studies are being encouraged to not only collect genetic information but to obtain detailed phenotype data-along with broader and more detailed information on environmental, behavioral, and lifestyle measures - in order to improve the capacity to link data sets for new kinds of analysis. Given the growing prevalence of a range of neurodevelopmental disorders in adults, there is an increasing interest collecting early cognitive, behavioral, metabolic, and epigenetic markers that might herald the onset and development of these diseases.

\subsubsection{Develop and Apply New and Improved Methodologies to Reflect a Complex Systems Approach and Enable Causal Inference}

As an emerging transdisciplinary field, LCHD research is typically done with scientific teams that include several methodologists and analysts to address the complex and multifaceted biological variation that epitomizes human health, but also the many and multilevel relational influences that are interacting and inducing changes in this continuously unfolding biological matrix. The complex and interconnected relationships of factors contributing to the development of obesity, mental health disorders, and other conditions with multiple complex causes typify the challenges that confound many common analytic approaches. Conventional longitudinal analyses, even those that account for multiple levels of influence, are often not powerful enough to account for the complexity and interconnectedness of obesity. Little's chapter in this volume (Little 2017) explains that several new techniques are available to improve estimation and causal inference for study designs with a multitude of time-varying covariates that interact across multiple levels. These include, among others, longitudinal structural equation modeling which can model hierarchically nested contextual influences on health over time. In addition, an emerging set of analytic tools from the systems sciences including agent-based and system dynamics modeling can be utilized to examine longitudinal relationships over multiple levels and, at the same time, account for more complex features of relationships like nonlinearity, path dependence, loops, and tipping (Hammond 2009; Huang, Drewnosksi, Kumanyika, and Glass 2009).

Several authors reference the need for better community-based participatory research involving collaboration and partnership between community leaders, residents, and academic researchers. This kind of research can be used to help design assessments of living conditions (i.e., 
risk factors and influences) that are richer in content and more appropriately reflect the complex interactions that take place in family and community settings. It can also help to design interventions that are responsive to local needs and promote policy advocacy in disadvantaged neighborhoods. As place-based efforts attempt to transform the basic conditions in a community, they have often taken a life course approach to improve cradle-to-career trajectories with attention paid to what works in different kinds of communities and microenvironments.

\subsubsection{Build, Strengthen, and Improve Institutional Capacity to Conduct LCHD Research}

Increasing our capacity to conduct life course health science research requires a systematic and purposeful effort that focuses on human skills and talent, infrastructure, and institutional capacity, as well as state-of-the-art life course research tools and methods. Capacity building at the individual level requires mentoring, career guidance, research opportunities, and research pathways that are transdisciplinary and designed to promote optimal development of a life course health development researcher. At an institutional level, advancing life course health science research requires an appreciation for its importance, as well as prioritization of resources allocated for integrative approaches that characterize LCHD research.

Current major research infrastructure building and reform efforts like the Clinical Translational Science Institutes (CTSIs) permit institutions to use life course health development as an integrating strategy for building cross-disciplinary research teams and for focusing research priorities on key health development strategies. The CTSIs have also encouraged approaching diseases and health conditions in a more holistic manner, moving beyond traditional clinic-based models to engage individuals and families in the context of their own lives and life course goals. Similarly, the Patient-Centered Outcome Research Initiative has spearheaded research from a patient's perspective that also lends itself to life course consideration, including challenges presented by specific life course transitions. Life course health science requires team-based, often multi-institutional approaches to research, which is rapidly becoming the norm in the natural, biomedical, and social sciences.

\subsection{Section III:Translational Priorities}

Efforts are needed to identify existing knowledge that is ready for translation to concrete practice guidelines, programs, and policies aimed at improving health trajectories and alleviating disparities in health. Bringing this life course health development lens to the current healthcare system permits a critical review of how medical care clinics are organized, how healthcare systems organize care pathways, and more. Perhaps the greatest practical clinical and programmatic realization coming from LCHD research is the value of care delivery pathways and systems that are purposeful in their horizontal integration across all of the social ecological domains that influence health development and that are inclusive of all of the sectors and potential influencers on the whole person's health. The other important integration strategy is one that focuses on longitudinal or life course integration, not only anticipating future needs and concerns but designed to optimize life course-organized transitions and challenges. For example, life course-oriented research focused on optimizing health development could be used to redesign the healthcare system with a greater emphasis on promoting optimal health throughout the life course from birth through death, instead of focusing on disease management alone (Halfon et al. 2014b, c). Continued efforts are needed to identify ways in which life course research on health disparities can be integrated into medical, social welfare, education, and public health practice, as well as social and health policies at the local, state, and national levels.

There is also a great need to begin to explore how different periods of the life course have been underappreciated for their potential influence and leverage on downstream outcomes. For example, 
the chapter in this volume by Del Giudice (2017) calls attention to the juvenile transition and how this underappreciated and virtually unexplored period may be an important developmental window for intervention. The author suggests that interventions focused on optimizing transitions and occurring during these grammar school years can have an important priming and long-term influence on adolescent development. The idea that intervening during a biological transition may afford more leverage to alter developmental trajectories resonates with recognizing the nonlinearity of developmental processes and their time sensitivity.

Several of the chapters focused on better screening and assessment tools and approaches that attempt to capture key emergent characteristics and needs so that families can be provided with information about the consequences of decisions being made for a child early in his or her life. Local, state, and national governments also need policies in place that enhance child health development throughout childhood.

Improving public health messaging regarding health development across the life course with specific messaging about healthy hearing trajectories, healthy growth and nutrition trajectories, healthy teeth, and healthy family trajectories can all begin to build awareness and insights into the importance of health development goals, pathways, and trajectories. These include providing growth standards to help individual families implement health-promoting activities, exercise standards, sleep standards, and the like. Such an effort could include both family- and practitionerbased education programs to promote more robust efforts surrounding nutrition and exercise recommendations.

Better ways of sharing life course health development information should also extend to communities so that the optimizing health development of children can be built into the community ethos and appropriate educational materials and policy initiatives developed. Many communities have launched initiatives to promote health development and well-being across the life course. For example, the city of Santa Monica, California, has a lifelong learning system in place and a well-being index that is designed to high- light life course specific health data (Warner and Kern 2013). These kinds of initiatives can promote the importance of life course health science and can mobilize ingenuity and resourcefulness at local and national levels.

\subsection{Section IV: Refinement of the LCHD Theoretical Framework}

As the chapter by Halfon and Forrest (2017) in this volume indicates, the LCHD framework is an initial version of what may become a new scientific paradigm. As such, LCHD is synthesizing research from a range of different disciplines in ways that will guide the development and testing of new theories of health development and organize future research based on principles established by the field. As the field matures, it will be important to update the framework and its principles, and to consider research approaches, methods, and questions that will further elaborate the framework, moving toward the creation of a model that helps guide future hypothesis-driven research. Important considerations are further developing the explanatory power of the overall framework and interrogating the veracity of the principles that structure the network of ideas, theories, hypotheses, and research questions.

Halfon and Forrest present seven principles that structure the contours of the LCHD framework. They have conceived of these principles not as a linear sequence of defining characteristics but as network of high-level, interconnected generalizations that are empirically supported, logically consistent, and holistically justified. The network presentation suggests that these different principles are distinct but not independent of each other and that judging their explanatory robustness often requires that we consider not the principle in isolation but in relationship to other principles. In keeping with our systems approach to understanding human health development, the system of interconnected and interdependent explanatory principles suggests a dynamic and flexible network of concepts that can be used to build and test theories of causation, interactions, and relational importance. Because some of the 
principles are not as well supported by a robust empirical literature, some priority should be given to focusing research that addresses areas where additional research is needed.

Further development of the LCHD framework, using it to define the scope and level of life course health development inquiry, and applying it as an organized approach to theory testing will all require continued knowledge synthesis. Because the LCHD framework incorporates a diverse set of factors which interact with each other over significant and variable time horizons, there is a growing need to more systematically analyze and synthesize this information with reference to the overall framework. Greater synthesis of existing cross-disciplinary knowledge of different aspects of LCHD is also necessary for more precise investigation of pathophysiologic mechanisms.

\subsubsection{The LCHD Framework, Precision Medicine, and Population Health}

As the chapter by Halfon and Forrest suggests, the LCHD framework grew out of recognition that health development is a complex adaptive response of a dynamic, multidimensional, and multilevel system comprising individuals and their contexts codeveloping over time. Adopting the holism of systems biology and other systems sciences, the LCHD framework places more emphasis on the relationship between different components of the system (holism) than on describing the component parts (reductionism). In keeping with emerging notions of precision medicine and precision population health, not only are the insights from systems biology important but also understanding and integrating panomics - the interdependent interactions between the genome, epigenome, microbiome, virome, and exposome. The dynamics, patterns, and predictability of these panomic relationships are not only important for better targeting cancer therapy or customizing the treatment of inflammatory bowel disease but also for creating more effective, efficient, and responsive prevention and health promotion strategies at the population level that are not based on simple notions of risk and dependent on one-size-fits-all interventions (Halfon 2016).
The LCHD framework not only provides a powerful process and tool for knowledge integration but importantly introduces novel and important considerations of emergent developmental processes and developmentally contingent time frames that are important for the synchronization and integration of particular influence, as well as the interactions, flexibility, and dynamics of these relationships across the different combined influences. As the healthcare system focuses more on precision medicine and precision population health, there will be a growing interest in better characterizing health development processes at the individual and population level.

As research using the LCHD framework gallops forward, attention should be paid to how to further develop this framework. Is there an emergent and adaptive strategy to enable the LCHD framework to grow, develop, mature, and evolve as the field of LCHD research moves forward? As LCHD evidence accumulates, is analyzed, and is synthesized, the number of LCHD principles may grow or shrink, and the relationship of the LCHD principles between the principles should become clearer and more useful as a more fully articulated LCHD paradigm emerges. Halfon and Forrest have conceptualized the principles as a network of conceptual nodes whose explanatory utility and robustness is only fully realized in relationship to each other. Modeling and more fully exploring the relationships between the principles can also help test whether this conceptualization of how the principles manifest has explanatory value. As an overarching meta-framework, it will also be useful to examine how the LCHD framework can inform new notions of precision medicine and emerging notions of precision population health.

\subsubsection{LCHD and Health System Transformation}

Halfon and Forrest also suggest that the LCHD framework is not only strategically responding to a large body of empirical work that challenges long held notions of the causal mechanism responsible for disease causation and the production of different levels of health, but that by focusing on how to optimize health development, it includes considerations of the complex health 
influencing ecosystem and how that health development ecosystem evolves and changes over time. The framework suggests that health development does not occur solely within an individual or even as a result of an individual's exposure to certain environmental factors, but rather results from continuous coactions between persons and their environments, which we have represented as $\mathrm{P} \Leftrightarrow \mathrm{E}$. Thus, individuals are affected by their environments, but they also shape their environments, and the focus on health development is on individual's health assets, their environmental influences, and the $\Leftrightarrow$ as well-that is, the relational mechanisms by which persons influence and are influenced by their environments (and vice versa). These insights not only challenge the simpler biomedical and biopsychosocial models of health and disease causation, but challenge the appropriateness, utility, effectiveness, and efficiency of a healthcare system that is largely built on a biomedical chassis and is just now in the process of upgrading its engine to accommodate psychosocial "upstream influences."

The LCHD framework implies that this new and more evolved paradigm of health development and disease causation provides the explanatory rational for how the healthcare delivery system evolves and is organized. The simple linear biomedical framework underwrote the rational for much of the 1.0 acute care focused medical care system with its focus on providing timelimited and rescue care for specific medical conditions over short time horizons. Similarly, the scientific advances and research that led to the development of the more dynamic and multilevel biopsychosocial framework begin to underwrite the upgrade and reengineering of the 2.0 chronic disease system with its greater focus on managing behavioral and lifestyle influences on chronic disease over longer time horizons. The emergence of the LCHD framework is already providing a way of underwriting the need for a more horizontally and longitudinally integrated, anticipatory, and developmentally primed health system (3.0 health system) that is structured to optimize health and health-promoting environments over the life span (Halfon et al. 2014b). Moving forward, it will be important to consider how the LCHD framework can be more fully exploited to inform healthcare innovations and health systems transformation. By fortifying a 3.0 operating system, can LCHD principles be translated into new healthcare system design concepts and innovation strategies? For example, does a better understanding of the potential vulnerability associated with the transition from homecare to preschool suggest a way in which services and supports should be developmentally organized to optimize this transition and support necessary adaptation on the part of the child as well as those in the family?

\subsubsection{The LCHD Framework and the Measurement of Health Development}

Health in the LCHD framework is defined as an emergent adaptive response to environmental (e.g., biological, behavioral, family, social, physical) challenges. The dynamics of health development and the emergence of diseases, dysfunction, or disintegration depend on dynamic processes and patterns of responses that typically span from the cellular to the whole person level. Because no component of this cluster of interdependent processes and purposeful relationships is ever standing still but is in a constant state of dynamic fluctuations, health is recognizable in the organism's dynamic developmental adaptability and their capacity to strategically respond to different environmental challenges. Because health is conditional on these different environments, measures of health development must be able to capture an individual and populations capacity to dynamically respond when an individual, population, or ecosystem is "out of balance." It also suggests the importance of capturing levels (cellular to ecosystem) of flexibility and resilience that are necessary and manifest to rebalance and achieve not only renewed level of health but an optimal level of health development. Our current measures of health and health development at an individual level are sorely lacking the capacity to sense and integrate signals from the cellular to the whole person level, across physiological systems and response patterns, and across developmentally significant time horizons. At a population level, there are similar conceptual and measurement gaps in how health devel- 
opment is measured and how to capture the developmentally contingent aspects of health development for different populations in facing different ecosystem contexts.

\section{Conclusions and Next Steps}

This volume has attempted to highlight the importance of the rapidly evolving field of life course health science and its potential impact on improving the lifelong health trajectories. We have used the life course health development framework as a way of organizing inquiry and understanding the relationship between a diverse but converging set of empirical findings. We have proposed a set of LCHD principles that are emerging and, we contend, on their way to describing a new paradigm of scientific investigation and can be used to organize and explain what has been learned and to guide and organize future inquiry (Ostrom 2009; Gatzweiler and Baumuller 2014). This volume has also begun to link and network related areas of inquiry, utilizing these common principles and what they illuminate about deeper and more complex processes of health development. Lastly, we have attempted to relate LCHD research to the field of maternal and child health and to explore how life course enlightened knowledge of health development can be used to improve the health of mothers and children and achieve greater health equity from the start of life.

In this volume, different teams of scholars have analyzed and synthesized the current state of knowledge and research progress in a range of important areas of health development. Spanning topics as diverse as hearing health, kidney health, metabolic health, and oral health across different developmental stages from preconception to emerging adulthood, the chapters examine the health development contribution of different life stages, revealing new considerations for how to prevent losses in health potential, as well as how to optimize lifelong health development. Through the process of writing their chapters, the authors have analyzed and synthesized what is currently known and have begun to shine a light on the way forward by identifying specific research questions and strategies intended to advance research productivity and impact, as well as translation to programs, policy, and practices.

\subsection{What's Next?}

There are several important national trends that make life course health science and future research on life course health development particularly salient and important at the present time (McGinnis et al. 2016). First are the growing concerns about persistent and structural inequalities in the USA. The life course health sciences have already contributed to the growing recognition of the lifelong health, social, and economic impacts of poverty-associated adversity early in life. Additional research focused on the impact of the early years on future health development will continue to play an important role in refocusing policy, programs, and practices on addressing and leveraging what is at stake in these early years (Halfon et al. 2014c).

Second are the opportunities provided by the implementation of the Affordable Care Act (ACA) and related initiatives designed to move healthcare upstream and to focus more attention and resources on prevention and population health. The life course health sciences are illuminating how such upstream strategies must focus not only on the social and environmental causes of health outcomes but in particular on the upstream influences on costly and preventable adult health outcomes (Wise 2016). As post-ACA policy attempts to steer healthcare financing from a focus on volume (outputs) to value (outcomes), there is a real opportunity to demonstrate how health development value chains begin early in life and how investments in health development capital can be leveraged into significant long-term value results (McGinnis et. al. 2016). ACA-stimulated health system reform is also accelerating the consideration of transformational strategies that are challenging the dominant 2.0 operating logic, structure, and organization that arose to address the diagnosis, treatment, and management of adult chronic health conditions. What is now emerging is a new 3.0 operating logic aimed at optimizing lifelong health by leveraging the new science of life course health development (Halfon et al. 2014b). The findings from the life course 
health sciences can continue to support this transition from 2.0 to 3.0 by providing the empirical basis for justifying healthcare systems that are more horizontally integrated to address multiple interacting dimensions and influences on health development, as well as longitudinally integrated to anticipate future health development scenarios and build long-term pull strategies into the structure and design of health and health-related social and educational services.

Third are the opportunities and challenges posed by major federal research initiatives, like those focused on precision medicine and clinical translational science and the lag from discovery to wide scale implementation. While both of these National Institutes of Health (NIH)-funded efforts begin with a biomedical orientation - with precision medicine focused on specifying the biological characteristics (phenotype) that will assist in pinpointing pharmacological interventions with greater accuracy and translational science focused on moving from the discovery of a new molecule in a laboratory and more rapidly and efficiently testing it at the hospital bedside and in the clinic making it widely available on a population basis-there is a critical need to inform these and other traditionally biomedically oriented research efforts with life course health science thinking and approaches.

Fourth is the fact that the national health policy focus on improving population health and reducing health disparities can benefit from the LCHD framework and life course health science results. As Larson and colleague (Larson et al. 2017) argue in this volume, "the LCHD framework emphasizes the importance of addressing disparities from preconception onward; understanding that early exposure to multiple stresses may produce biological changes that are difficult, if not impossible, to eradicate later; and acknowledging that adults who experienced the most extreme disparities as young children are likely to develop the worst health in adulthood and require the most expensive interventions. Life course-oriented health disparities research provides an opportunity for large potential savings associated with the development of strategies to intervene early in optimizing human health." Real headway in not only addressing health dis- parities but reducing these inequalities in health development will arise by taking advantage of the life course health science along with the opportunities afforded by ACA-induced health system transformation to focus greater attention and the role of the early years in reducing disparities, decreasing the prevalence and impact of chronic illness, and saving healthcare costs.

As a product of the Life Course Research Network (LCRN) that is funded by the Department of Health and Human Services (DHHS) Health Resources and Services Administration (HRSA) Maternal and Child Health Bureau (MCHB), this volume is meant - in addition to serving as a catalyst and tool for advancing the LCHD field - to inform MCHB's thinking, planning, and funding of future research. To this end, the content of this chapter will be used to create a more traditionally structured research agenda containing specific research questions that the members of the LCRN (see lcrn.net to learn more and join) and the broader community of LCHD stakeholders will be invited to participate in refining and prioritizing among. Over time, the LCRN-which was recently awarded another 3 years of funding from $\mathrm{MCHB}$ - will produce, commission and/ or solicit additional research agenda-setting papers on topics missing from the present volume in order to enable the periodic updating of the LCRA and enhance the dissemination and synthesis of emerging findings. Taken together, these efforts will help to advance both the life course health sciences and our understanding of their importance for optimizing life course health development.

\section{References}

Barker, D. J., Osmond, C., Winter, P. D., Margetts, B., \& Simmonds, S. J. (1989). Weight in infancy and death from ischaemic heart disease. The Lancet, 334(8663), 577-580.

Ben-Shlomo, Y., \& Kuh, D. (2002). A life course approach to chronic disease epidemiology: Conceptual models, empirical challenges and interdisciplinary perspectives. International Journal of Epidemiology, 31(2), 285-293.

Birbeck, G. L., Meyer, A.-C., \& Ogunniyi, A. (2015). Nervous system disorders across the life course in resource-limited settings. Nature, 527(7578), S167-S171. 
Boyce, W. T., \& Hertzman, C. (2017). Early childhood and the life course: The state of the science and proposed research priorities. In N. Halfon, C. B. Forrest, R. M. Lerner, \& E. Faustman (Eds.), Handbook of life course health-development science. Cham: Springer.

Brophy, P. D., Charlton, J. R., Carmody, J. B., Reidy, K. J., Harshman, L., Segar, J., Askenazi, D., \& Shoham, D. (2017). Chronic kidney disease: A life course health development perspective. In N. Halfon, C. B. Forrest, R. M. Lerner, \& E. Faustman (Eds.), Handbook of life course health-development science. Cham: Springer.

Brownell, M. D., Ekuma, O., Nickel, N. C., Chartier, M., Koseva, I., \& Santos, R. G. (2016). A populationbased analysis of factors that predict early language and cognitive development. Early Childhood Research Quarterly, 35, 6-18.

Buka, S. L., Rosenthal, S. R., \& Lacy, M. E. (2017). Epidemiological study designs: Traditional and novel approaches to advance life course health development research. In N. Halfon, C. B. Forrest, R. M. Lerner, \& E. Faustman (Eds.), Handbook of life course healthdevelopment science. Cham: Springer.

Cohen, A. A. (2016). Complex systems dynamics in aging: New evidence, continuing questions. Biogerontology, 17(1), 205-220.

Cooksey, E. (2017). Using the National Longitudinal Surveys of Youth (NLSY) to conduct life course analyses. In N. Halfon, C. B. Forrest, R. M. Lerner, \& E. Faustman (Eds.), Handbook of life course healthdevelopment science. Cham: Springer.

Currie, J., \& Stabile, M. (2006). Child mental health and human capital accumulation: The case of ADHD. Journal of Health Economics, 25(6), 1094-1118.

Davidson, L. L., Grigorenko, E. L., Boivin, M. J., Rapa, E., \& Stein, A. (2015). A focus on adolescence to reduce neurological, mental health and substance-use disability. Nature, 527(7578), S161-S166.

DelGiudice, M. (2017). Middle childhood: An evolutionary-developmental synthesis. In N. Halfon, C. B. Forrest, R. M. Lerner, \& E. Faustman (Eds.), Handbook of life course health-development science. Cham: Springer.

Drury, S. S., Theall, K., Gleason, M. M., Smyke, A. T., De Vivo, I., Wong, J. Y., Fox, N. A., Zeanah, C. H., \& Nelson, C. A. (2012). Telomere length and early severe social deprivation: Linking early adversity and cellular aging. Molecular Psychiatry, 17(7), 719-727.

Duncan, G. J., Morris, P. A., \& Rodrigues, C. (2011). Does money really matter? Estimating impacts of family income on young children's achievement with data from random-assignment experiments. Developmental Psychology, 47(5), 1263.

Dunn, E. C., Masyn, K. E., Yudron, M., Jones, S. M., \& Subramanian, S. V. (2014). Translating multilevel theory into multilevel research: Challenges and opportunities for understanding the social determinants of psychiatric disorders. Social Psychiatry and Psychiatric Epidemiology, 49(6), 859-872.

Dunn, E. C., Richmond, T. K., Milliren, C. E., \& Subramanian, S. V. (2015). Using cross-classified multilevel models to disentangle school and neighborhood effects: An example focusing on smoking behaviors among adolescents in the United States. Health \& Place, 31, 224-232.

El-Sayed, A. M., Seemann, L., Scarborough, P., \& Galea, S. (2013). Are network-based interventions a useful antiobesity strategy? An application of simulation models for causal inference in epidemiology. American Journal of Epidemiology, 178(2), 287-295.

Feinberg, M. E., Kan, M. L., \& Goslin, M. C. (2009). Enhancing coparenting, parenting, and child selfregulation: Effects of family foundations 1 year after birth. Prevention Science, 10(3), 276-285.

Felitti, V. J., Anda, R. F., Nordenberg, D., Williamson, D. F., Spitz, A. M., Edwards, V., Koss, M. P., \& Marks, J. S. (1998). Relationship of childhood abuse and household dysfunction to many of the leading causes of death in adults: The adverse childhood experiences (ACE) study. American Journal of Preventive Medicine, 14(4), 245-258.

Gatzweiler, F. S., \& Baumuller, H. (2014). Marginality-a framework for analyzing causal complexity of poverty. In J. von Braun \& F. W. Gatzweiler (Eds.), Marginality: Addressing the nexus of poverty, exclusion, and ecology. Dordrecht: Springer.

Geller, A., Jaeger, K., \& Pace, G. (2017). Using the Fragile Families and Child Wellbeing Study (FFCWS) in life course health development research. In N. Halfon, C. B. Forrest, R. M. Lerner, \& E. Faustman (Eds.), Handbook of life course health-development science. Cham: Springer.

Guhn, M., Gadermann, A. M., Almas, A., Schonert-Reichl, K. A., \& Hertzman, C. (2016a). Associations of teacherrated social, emotional, and cognitive development in kindergarten to self-reported wellbeing, peer relations, and academic test scores in middle childhood. Early Childhood Research Quarterly., 35, 76-84.

Guhn, M., Janus, M., Enns, J., Brownell, M., Forer, B., Duku, E., Muhajarine, N., \& Raos, R. (2016b). Examining the social determinants of children's developmental health: Protocol for building a pan-Canadian population-based monitoring system for early childhood development. BMJ Open, 6(4), e012020.

Halfon, N. (2016). More precisely targeting the coal mine of social adversity. JAMA Pediatrics, 170(11), e162523.

Halfon, N., \& Forrest, C. B. (2017). The emerging theoretical framework of life course health development. In N. Halfon, C. B. Forrest, R. M. Lerner, \& E. Faustman (Eds.), Handbook of life course health-development science. Cham: Springer.

Halfon, N., Larson, K., \& Slusser, W. (2013). Associations between obesity and comorbid mental health, developmental, and physical health conditions in a nationally representative sample of US children aged 10 to 17. Academic Pediatrics, 13(1), 6-13.

Halfon, N., Larson, K., Lu, M., Tullis, E., \& Russ, S. (2014a). Lifecourse health development: Past, present and future. Maternal and Child Health Journal, 18(2), 344-365. 
Halfon, N., Long, P., Chang, D. I., Hester, J., Inkelas, M., \& Rodgers, A. (2014b). Applying a 3.0 transformation framework to guide large-scale health system reform. Health Affairs, 33(11), 2003-2011.

Halfon, N., Wise, P. H., \& Forrest, C. B. (2014c). The changing nature of children's health development: New challenges require major policy solutions. Health Affairs (Millwood)., 33(12), 2116-2124.

Hammond, R. A. (2009). Peer reviewed: complex systems modeling for obesity research. Preventing Chronic Disease, 6(3), A97.

Hane, A. A., \& Fox, N. A. (2016). Early caregiving and human biobehavioral development: A comparative physiology approach. Current Opinion in Behavioral Sciences, 7, 82-90.

Hanson, M. A., \& Gluckman, P. D. (2014). Early developmental conditioning of later health and disease: Physiology or pathophysiology? Physiological Reviews, 94(4), 1027-1076.

Hawkins, S. S., Oken, E., \& Gillman, M. W. (2017). Early in the life course: Time for obesity prevention. In N. Halfon, C. B. Forrest, R. M. Lerner, \& E. Faustman (Eds.), Handbook of life course health-development science. Cham: Springer.

Herman, D., \& Taylor-Baer, M. (2017). From epidemiology to epigenetics: Evidence for the importance of nutrition to optimal health development across the life course. In N. Halfon, C. B. Forrest, R. M. Lerner, \& E. Faustman (Eds.), Handbook of life course healthdevelopment science. Cham: Springer.

Hertzman, C., \& Boyce, T. (2010). How experience gets under the skin to create gradients in developmental health. Annual Review of Public Health, 31, 329-347.

Hochberg, Z.'e. (2009). Evo-devo of child growth II: Human life history and transition between its phases. European Journal of Endocrinology, 160(2), 135-141.

Huang, T. T., Drewnowski, A., Kumanyika, S. K., \& Glass, T. A. (2009). A systems-oriented multilevel framework for addressing obesity in the 21 st century. Preventing Chronic Disease, 6(3), A82.

Janus, M., Harrison, L. J., Goldfeld, S., Guhn, M., \& Brinkman, S. (2016). International research utilizing the early development instrument (EDI) as a measure of early child development: Introduction to the special issue. Early Childhood Research Quarterly., $35,1-5$.

Karatsoreos, I. N., \& McEwen, B. S. (2013). Annual research review: The neurobiology and physiology of resilience and adaptation across the life course. Journal of Child Psychology and Psychiatry, 54(4), 337-347.

Kim, P., Strathearn, L., \& Swain, J. E. (2016). The maternal brain and its plasticity in humans. Hormones and Behavior, 77, 113-123.

Kim, P., Evans, G. W., Chen, E., Miller, G., \& Seeman, T. (2017). How socioeconomic disadvantages get under the skin and into the brain to influence healthdevelopment across the lifespan. In N. Halfon, C. B. Forrest, R. M. Lerner, \& E. Faustman (Eds.), Handbook of life course health-development science. New York: Springer.
Kitano, H. (2004). Biological robustness. Nature Reviews Genetics, 5, 826-837.

Kuh, D., \& Shlomo, Y. B. (2004). A life course approach to chronic disease epidemiology. Oxford: Oxford University Press.

Larson, K., Russ, S. A., Kahn, R. S., Flores, G., Goodman, E., Cheng, T. L., \& Halfon, N. (2017). Health disparities: A life course health development perspective and future research directions. In N. Halfon, C. B. Forrest, R. M. Lerner, \& E. Faustman (Eds.), Handbook of life course health-development science. Cham: Springer.

Lerner, R. M., Brindis, C. C., Batanova, M., \& Blum, R. W. (2017). Adolescent health: A relational developmental systems perspective. In N. Halfon, C. B. Forrest, R. M. Lerner, \& E. Faustman (Eds.), Handbook of life course health-development science. Cham: Springer.

Leslie, L. K., Mehus, C. J., Hawkins, J. D., Boat, T., et al. (2016). Primary care: Potential home for family focused interventions. American Journal of Preventive Medicine, 51(4), S106-S118.

Little, T. D. (2017). Core principles of life course health development methodology and analytics. In N. Halfon, C. B. Forrest, R. M. Lerner, \& E. Faustman (Eds.), Handbook of life course health-development science. Cham: Springer.

McGinnis, J. M., Diaz, A., \& Halfon, N. (2016). Systems strategies for health throughout the life course. JAMA, 316(16), 1639-1640.

Merikangas, K. R., He, J. P., Burstein, M., Swanson, S. A., Avenevoli, S., Cui, L., Benjet, C., Georgiades, K., \& Swendsen, J. (2010). Lifetime prevalence of mental disorders in US adolescents: Results from the National Comorbidity Survey Replication-Adolescent Supplement (NCS-A). Journal of the American Academy of Child and Adolescent Psychiatry, 49(10), 980-989.

Moffitt, T. E. (1993). Adolescence-limited and lifecourse-persistent antisocial behavior: A developmental taxonomy. Psychological Review, 100(4), 674.

Msall, M. E., Sobotka, S. A., Dmowska, A., Hoga, D., \& Sullivan, M. (2017). Life course health development outcomes after prematurity: Developing a community, clinical, and translational research agenda to optimize health, behavior and functioning. In N. Halfon, C. B. Forrest, R. M. Lerner, \& E. Faustman (Eds.), Handbook of life course health-development science. Cham: Springer.

Mummert, A., Schoen, M., \& Lampl, M. (2017). Growth and life course health development. In N. Halfon, C. B. Forrest, R. M. Lerner, \& E. Faustman (Eds.), Handbook of life course health-development science. Cham: Springer.

Ostrom, E. (2009). A general framework for analyzing sustainability of social-ecological systems. Science, 325(5939), 419-422.

Osypuk, T. L. (2013). Invited commentary: Integrating a life-course perspective and social theory to advance research on residential segregation and health. American Journal of Epidemiology, 177(4), 310-315.

Patterson, M. L., Moniruzzaman, A., \& Somers, J. M. (2015). History of foster care among homeless 
adults with mental illness in Vancouver, British Columbia: A precursor to trajectories of risk. BMC Psychiatry, 15(1), 1.

Pearce, J. (2014). Invited commentary: history of place, life course, and health inequalities - historical geographic information systems and epidemiologic research. American Journal of Epidemiology, 181(1), 26-29.

Pearce, J., Shortt, N., Rind, E., \& Mitchell, R. (2016). Life course, green space and health: Incorporating place into life course epidemiology. International Journal of Environmental Research and Public Health, 13(3), 331.

Robinson, O., Basagaña, X., Agier, L., et al. (2015). The pregnancy exposome: Multiple environmental exposures in the INMA-Sabadell birth cohort. Environmental Science \& Technology, 49(17), 10632-10641.

Russ, S. A., Tremblay, K., Halfon, N., \& Davis, A. (2017). A life course approach to hearing health. In N. Halfon, C. B. Forrest, R. M. Lerner, \& E. Faustman (Eds.), Handbook of life course health-development science. Cham: Springer.

Sastry, N., Fomby, P., \& McGonagle, K. (2017). Using the Panel Study of Income Dynamics (PSID) to conduct life course health development analysis. In N. Halfon, C. B. Forrest, R. M. Lerner, \& E. Faustman (Eds.), Handbook of life course health-development science. Cham: Springer.

Schonert-Reichl, K. A., Guhn, M., Gadermann, A. M., Hymel, S., Sweiss, L., \& Hertzman, C. (2013). Development and validation of the middle years development instrument (MDI): Assessing children's well-being and assets across multiple contexts. Social Indicators Research, 114(2), 345-369.

Silberberg, D., Anand, N. P., Michels, K., \& Kalaria, R. N. (2015). Brain and other nervous system disorders across the lifespan [mdash] global challenges and opportunities. Nature, 527(7578), S151-S154.

Singer, B. H., \& Ryff, C. D. (Eds.). (2001). New horizons in health: An integrative approach (Vol. 277). Washington, D.C.: National Academies Press.
Taveras, E. M., Blackburn, K., Gillman, M. W., Haines, J., McDonald, J., Price, S., \& Oken, E. (2011a). First steps for mommy and me: a pilot intervention to improve nutrition and physical activity behaviors of postpartum mothers and their infants. Maternal and Child Health Journal, 15(8), 1217-1227.

Taveras, E. M., Gortmaker, S. L., Hohman, K. H., Horan, C. M., Kleinman, K. P., Mitchell, K., \& Gillman, M. W. (2011b). Randomized controlled trial to improve primary care to prevent and manage childhood obesity: The high five for kids study. Archives of Pediatrics \& Adolescent Medicine, 165(8), 714-722.

Taveras, E. M., McDonald, J., O’Brien, A., Haines, J., Sherry, B., Bottino, C. J., \& Koziol, R. (2012). Healthy Habits, Happy Homes: Methods and baseline data of a randomized controlled trial to improve household routines. Preventive Medicine, 55(5), 418-426.

Tyler, K. A., \& Schmitz, R. M. (2013). Family histories and multiple transitions among homeless young adults: Pathways to homelessness. Children and Youth Services Review, 35(10), 1719-1726.

Warner, K., \& Kern, M. (2013, December). A City of Wellbeing: The what, why \& how of measuring community wellbeing. The City of Santa Monica Office of Wellbeing.

Wise, P. H. (2016). Child poverty and the promise of human capacity: Childhood as a foundation for healthy aging. Academic Pediatrics, 16(3), S37-S45.

Wood, D., Crapnell, T., Lau, L., Bennett, A., Lotstein, D., Ferris, M., \& Kuo, A. (2017). Emerging adulthood as a critical stage in the life course. In N. Halfon, C. B. Forrest, R. M. Lerner, \& E. Faustman (Eds.), Handbook of life course health-development science. Cham: Springer.

Zlotnick, C., Tam, T. W., \& Soman, L. A. (2012). Life course outcomes on mental and physical health: The impact of foster care on adulthood. American Journal of Public Health, 102(3), 534-540.

Open Access This chapter is licensed under the terms of the Creative Commons Attribution 4.0 International License (http://creativecommons.org/licenses/by/4.0/), which permits use, sharing, adaptation, distribution and reproduction in any medium or format, as long as you give appropriate credit to the original author(s) and the source, provide a link to the Creative Commons license and indicate if changes were made.

The images or other third party material in this chapter are included in the chapter's Creative Commons license, unless indicated otherwise in a credit line to the material. If material is not included in the chapter's Creative Commons license and your intended use is not permitted by statutory regulation or exceeds the permitted use, you will need to obtain permission directly from the copyright holder. 